\title{
Retraction Note to: Correlation of IL-1F genetic polymorphisms with the risk of colorectal cancer among Chinese populations
}

\author{
Ming Ma ${ }^{1}$ Guo-Jiang Jin ${ }^{1} \cdot$ Ke Yun ${ }^{1} \cdot$ Run-Qing Mu ${ }^{1} \cdot$ Min Zhao ${ }^{1}$ Xiao-Ou Yu ${ }^{1}$. \\ Shuo Wang ${ }^{1} \cdot$ Hong Shang ${ }^{1}$
}

Published online: 17 August 2015

(C) International Society of Oncology and BioMarkers (ISOBM) 2015

Retraction Note to: Tumor Biol. (2015) 36:807-814

DOI 10.1007/s13277-014-2653-y

The Publisher and Editor retract this article in accordance with the recommendations of the Committee on Publication Ethics (COPE). After a thorough investigation we have strong reason to believe that the peer review process was compromised.

The online version of the original article can be found at http://dx.doi.org/ 10.1007/s13277-014-2653-y.

\footnotetext{
Hong Shang

hongshang100@hotmail.com
}

1 Department of Laboratory Medicine, The First Hospital of China, Medical University, Nanjing Street No. 155, Heping District,

Shenyang 110000, People's Republic of China 